\title{
Increasing Naloxone Co-prescription for Patients on Chronic Opioids: a Student-Led Initiative
}

\author{
Jonathan E. Freise, BS, $M S^{7}$, Elizabeth E. McCarthy, $B S^{7}$, Michelle Guy, $M D^{1,2}$, Scott Steiger, $M D^{1,2}$, \\ and Leslie Sheu, MD ${ }^{1,2}$
}

${ }^{1}$ School of Medicine, University of California, San Francisco, San Francisco, CA, USA; ${ }^{2}$ Department of Medicine, University of California, San Francisco, San Francisco, CA, USA.

KEY WORDS: naloxone; opioids; primary care; quality improvement; medical education.

J Gen Intern Med 33(6):797-8

DOI: $10.1007 / \mathrm{s} 11606-018-4397-7$

(c) Society of General Internal Medicine 2018

\section{INTRODUCTION}

In 2016, the Center for Disease Control (CDC) established guidelines for primary care providers prescribing opioids for chronic pain, including naloxone co-prescription for patients on high-dose chronic opioids (morphine equivalent daily dose $(\mathrm{MED}) \geq 50 \mathrm{mg}){ }^{1}$

Despite naloxone's proven efficacy, naloxone coprescription in primary care settings remains low. ${ }^{2}$ This student-led quality improvement effort assessed barriers to naloxone co-prescription at an academic primary care clinic and implemented and evaluated a targeted intervention to increase naloxone co-prescription for patients on high-dose chronic opioids for non-cancer pain.

\section{METHODS}

As part of a new medical school pre-clerkship curriculum, first-year medical students were paired with faculty to conduct quality improvement projects within the faculty members' clinical setting. We aimed to increase naloxone coprescription for a registry of patients prescribed chronic opioids within the University of California, San Francisco (UCSF), Division of General Internal Medicine, an academic primary care clinic.

In a needs assessment survey with multiple-choice questions, providers were asked about barriers to prescribe naloxone by selecting all answers that applied (out of eight possible choices). Forty-nine of 117 (42\%) providers responded, and the two most commonly reported barriers were lack of provider comfort (41\%) and lack of time (59\%), consistent with previous studies. $^{3}$ To increase provider comfort, we sent individualized emails to all providers who had patients in the

Jonathan E. Freise and Elizabeth E. McCarthy contributed equally to this work.

Published online March 19, 2018 registry $(N=101)$, including each patient's MED, naloxone co-prescription status, and a suggested script for discussing naloxone with patients based on previous recommendations (Text Box). ${ }^{4}$ To address time burden, we provided medical assistants (MAs) with a list of patients on high-dose chronic opioids without a naloxone co-prescription and an upcoming clinic appointment every 2 weeks during the intervention period (October 2016-February 2017). MAs queued naloxone prescription orders in the electronic medical record (EMR) during the check-in process to serve as visual reminders for providers to discuss naloxone and to reduce the time for prescribing naloxone. If desired, providers could complete an order for "naloxone counseling" to prompt licensed vocational nurses (LVNs) trained in naloxone counseling to teach patients how to use the medication. Patient education lasted 5$10 \mathrm{~min}$, including watching an informational video and a demonstration using a sample of naloxone.

Text box

A sample individualized email sent to providers at the clinic.

How should I discuss a naloxone prescription with my patient?

A great time to bring up a naloxone prescription is while you are checking in about pain management with your patient's current pain medication. Recent high-profile cases of accidental opioid overdoses (e.g., Prince) can be a great starting point for talking about the dangers of accidental overdose with opioids.

When suggesting a naloxone prescription, you can remind patients that naloxone is a safety measure not just for them but also for family members or friends who might take their opioids. It is helpful to use phrases such as "bad reaction" and "slows or stops breathing" because patients prescribed chronic opioids do not usually consider themselves to be at risk of an overdose. This can also be a lead into educating your patient about the dangers associated with chronic opioid use and suggesting alternative pain management strategies.

One notable best practice for naloxone prescription is to suggest that an at-risk patient create a "bad reaction plan" to share with friends, partners, and/or caregivers. Such a plan would contain information on the signs of overdose and how to administer naloxone or otherwise provide emergency care (as by calling 911).

Our primary outcome was the proportion of patients with naloxone co-prescriptions before and after our intervention. We performed a negative binomial regression to determine the effect of our intervention on the monthly number of naloxone co-prescriptions in the clinic. We also report the number of monthly naloxone co-prescriptions after completion of our intervention. Institutional review board approval by the UCSF 


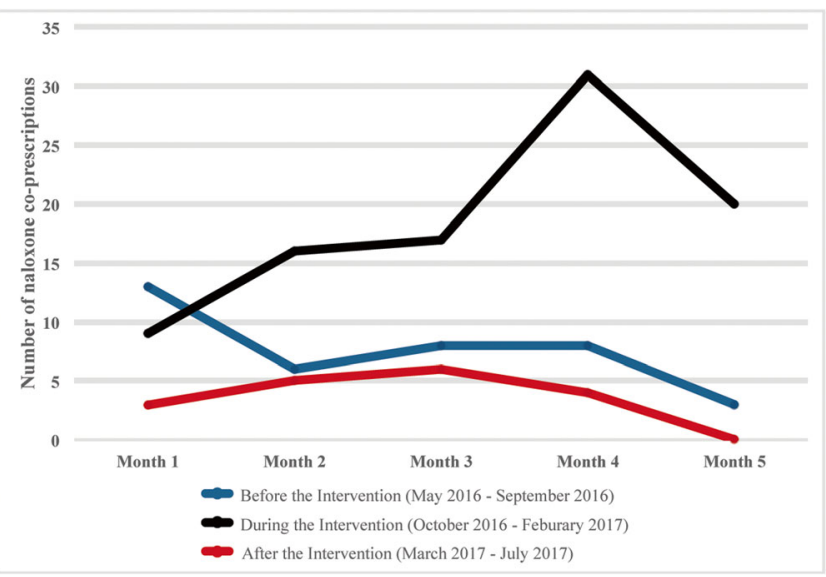

Figure 1 Number of naloxone co-prescriptions for patients on highdose chronic opioids each month during 5-month periods before, during, and after our intervention.

Committee on Human Research was not required because this was deemed a quality improvement project. $^{5}$

\section{RESULTS}

The proportion of naloxone co-prescriptions among patients on high-dose chronic opioids increased from $28 \%$ (76/271) before the intervention to $56 \%(146 / 262)$ after the intervention. We determined a statistically significant relationship between the number of monthly naloxone co-prescriptions and our intervention (incidence rate ratio (IRR) 2.45, 95\% CI 1.46 to 4.11). During the period following our intervention (March 2017-July 2017), the number of monthly naloxone coprescriptions declined (Fig. 1).

\section{DISCUSSION}

Our study provided an indication that a student-led local needs assessment and a targeted interprofessional team-based intervention, in the context of national urgency in the opioid overdose epidemic, may have improved naloxone coprescription among patients on high-dose chronic opioids. One limitation of our analysis is that a small number of patients may have had appointments both before and during, or during and after, our intervention, but this likely had little impact on our primary outcome. Despite the success of our intervention, after our intervention, the number of monthly naloxone co-prescriptions declined suggesting that enthusiastic stakeholders (medical students) rallying the team in the effort, as well as MAs queuing naloxone orders prior to patient encounters with providers may have been key components of this intervention. Possible future approaches include naloxone co-prescription as a requirement in patient-provider treatment agreements, automated reports on naloxone prescribing practices, and integrating automatic EMR reminders for prescribing naloxone. Confirming whether patients fill or refill their naloxone prescriptions and addressing other patient barriers might also improve patient safety.

Corresponding Author: Leslie Sheu, MD; Department of Medicine University of California, San Francisco, San Francisco, CA, USA (e-mail: leslie.sheu@ucsf.edu).

Funding Information The statistical analysis consultation for this project was supported by the National Center for Advancing Translational Sciences, National Institutes of Health, through UCSF-CTSI Grant Number UL1 TROO1872. Its contents are solely the responsibility of the authors and do not necessarily represent the official views of the NIH.

\section{Compliance with Ethical Standards:}

Conflict of Interest: The authors declare that they have no conflict of interest.

\section{REFERENCES}

1. Dowell D, Haegerich TM, Chou R. CDC guideline for prescribing opioids for chronic pain-United States, 2016. MMWR Recomm Rep. 2016;65:149.

2. Mundin, G., McDonald, R., Smith, K., Harris, S., and Strang, J. (2017) Pharmacokinetics of concentrated naloxone nasal spray over first 30 minutes post-dosing: analysis of suitability for opioid overdose reversal. Addiction, 112: 1647-1652.

3. Behar E. Acceptability of naloxone co-prescription among primary care providers treating patients on long-term opioid therapy for pain. J Gen Intern Med. 2017;32(3):291-95.

4. Starrels JL. Capsule commentary on mueller et al., attitudes toward naloxone prescribing in clinical settings: a qualitative study of patients prescribed high dose opioids for chronic non-cancer pain. J Gen Intern Med. 2017;32:312.

5. UCSF Institutional Review Board. Quality improvement (QI) and Quality Assurance (QA). Available at: https://irb.ucsf.edu/quality-improvementqi-and-quality-assurance-qa. Accessed January 8, 2018. 